\title{
Utilização da toxina botulínica tipo a para fins terapêuticos
}

\author{
Use of botulinum toxin type a for therapeutic purposes \\ Uso de toxina de botulinio tipo a con fines terapêuticos
}

Recebido: 27/10/2021 | Revisado: 05/11/2021 | Aceito: 08/11/2021 | Publicado: 14/11/2021

\section{Resumo}

Maressa Lima Da Silva

ORCID: https://orcid.org/0000-0001-7033-2922 Instituto Educacional Santa Catarina, Brasil E-mail: maressalima01@gmail.com

Letícia Ramos Ferreira

ORCID: https://orcid.org/0000-0002-9029-5556 Instituto Educacional Santa Catarina, Brasil E-mail: leticiaramoos7@gmail.com

Ana Carla Peixoto

ORCID: https://orcid.org/0000-0002-7285-8239 Instituto Educacional Santa Catarina, Brasil E-mail: anacarla_peixoto@hotmail.com

Liberta Lamarta Favoritto Garcia Neres ORCID: https://orcid.org/0000-0003-1327-605X Instituto Educacional Santa Catarina, Brasil E-mail: liberta.neres@iescfag.edu.br

Mara Régina Lucena Cabral

ORCID: https://orcid.org/0000-0003-2739-7231 Instituto Educacional Santa Catarina, Brasil E-mail: mararegina.uft@gmail.com

O trabalho tem por objetivo demonstrar a importância da toxina botulínica no tratamento da dor apresentando a necessidade de uma análise descritiva para a importância da aplicação e seus respectivos mecanismos de ação, buscando um levantamento em artigos acadêmicos da ANVISA, CDL e SCIELO nos anos entre 1980 a 2014, sendo a mais procurada para uso na estética poucas pessoas tem o conhecimento que ela pode trazer benefícios também para aplicações nas dores. A aplicação da TBA é um tratamento na qual varia de acordo com a extensão e a força no musculo a ser tratado, quanto maior for a força do musculo ou mais extenso, maior será a dose, sendo assim o tratamento com a TBA além de ter um elevado grau de satisfação também tem um índice baixo em relação as complicações ou efeitos colaterais.

Palavras-chave: Toxina botulínica; Mialgia; Clostridium botulinum.

\begin{abstract}
The work aims to demonstrate the importance of botulinum toxin in the treatment of pain, presenting the need for a descriptive analysis for the importance of the application and its respective mechanisms of action, seeking a survey in academic articles from ANVISA, CDL and SCIELO in the 1980s a 2014, being the most sought after for use in aesthetics, few people are aware that it can also bring benefits for pain applications. The application of TBA is a treatment in which it varies according to the extension and strength of the muscle to be treated, the greater the strength of the muscle or more extensive, the higher the dose, so the treatment with TBA in addition to having a high degree of satisfaction also has a low rate of complications or side effects.
\end{abstract}

Keywords: Botulinum toxin; Myalgia; Clostridium botulinum.

\section{Resumen}

El trabajo tiene como objetivo demostrar la importancia de la toxina botulínica en el tratamiento del dolor, presentando la necesidad de un análisis descriptivo de la importancia de la aplicación y sus respectivos mecanismos de acción, buscando una encuesta en artículos académicos de ANVISA, CDL y SCIELO en el 1980 a 2014, siendo el más buscado para su uso en estética, pocas personas son conscientes de que también puede traer beneficios para aplicaciones de dolor. La aplicación de TBA es un tratamiento en el que varía según la extensión y fuerza del músculo a tratar, a mayor fuerza del músculo o más extenso, mayor es la dosis, por lo que el tratamiento con TBA además de tener un alto grado de satisfacción también tiene una baja tasa de complicaciones o efectos secundarios.

Palabras clave: Toxina botulínica; Mialgia; Clostridium botulinum. 


\section{Introdução}

A toxina botulínica (TB) é produzida pelas bactérias Clostridium botulinum e Gram Anaeróbio positivo, esporulação. A história do Botox começou no final do século 18 e foi supostamente envenenado depois de comer linguiça de sangue e linguiça de carne, com isso aconteceram várias mortes no Reino de Württemberg, Alemanha (Romero et all, 2020).

A formação de esporos desse microrganismo produz toxinas que causam o botulismo, síndrome que causa diplopia embaçada, pálpebras caídas e fala arrastada, dificuldade em engolir, boca seca e fraqueza muscular. O botulismo infantil é ainda mais notório no mundo, bebês com botulismo sentem perda de apetite, letargia, prisão de ventre e diminuição dos tônus muscular, sintomas aqueles que desenvolvem paralisia muscular se não forem tratados podendo desenvolver a paralisia dos músculos respiratórios, braços, pernas e tronco e morte (Münchau, 2000).

A Toxina botulínica é considerada uma das substâncias mais mortais conhecidas hoje. Os esporos do Botox podem ser encontrados em feijões, vegetais, solo, as fezes humanas e intestinos de bovinos e pássaros são principalmente no mel. Há três formas de botulismo, derivado de alimentos ou através de uma ferida está contaminada com bactérias.

Existem sete tipos diferentes desta toxina, a saber: A, B, C ( $\mathrm{c}^{1}$ e c $\left.\mathrm{c}^{2}\right)$, D, E, F e G. Os tipos A, B, E e F são as causas mais comuns de botulismo. Existem terapias de espasmo, dor e terapia de beleza, a mais comumente usada é o tipo A, seu efeito é mais forte que outros tipos, pode ser tomado de acordo com a dosagem menor. Embora a toxina A seja amplamente utilizada, a toxina B também segue para fins estéticos (Rowlands et all, 2010).

O uso da TB para fins estéticos começou na década de 1990, quando observou que algumas rugas dos pacientes tratados melhoraram espasmo da pálpebra. E também relatou a melhora da atenuação da linha de expressão unilateral em pacientes recebendo tratamento para espasticidade para espasmos hemifaciais. O uso de toxinas do tipo A provou ser um método seguro e maduro para tratamento cosmético de rugas (Truong dd, 2009).

O principal mecanismo de ação da TB é bloquear a acetilcolina na junção neuromuscular, mas sem alterar a transmissão neuronal dos sinais elétricos e sem alterar a produção e armazenamento da acetilcolina, de modo que esse mecanismo é capaz de paralisar os músculos. No entanto, há estudos que mostram que a toxina botulínica atua bloqueando outras substâncias, como

o glutamato, a substância P e o peptídeo relacionado ao gene da calcitonina. Essas substâncias estão envolvidas em processos inflamatórios e causam sensibilização dos nervos, culminando em condições dolorosas (Ribeiro et al, 2014).

Esses mecanismos de bloqueio explicam os efeitos da toxina botulínica na redução / eliminação de condições dolorosas. A aplicação intramuscular da toxina em local adequado causa desenervação química parcial e redução da contratura sem causar paralisia completa. (Ribeiro et al, 2014).

Nosso estudo teve como objetivo revisar a literatura científica para destacar os avanços significativos no uso da toxina botulínica no tratamento de síndromes dolorosas, examinar seu mecanismo de ação e os resultados bem-sucedidos no tratamento dessas síndromes.

\section{Metodologia}

Esta pesquisa tem como objetivo explicitar e apresentar hipóteses acerca do problema apresentado, aprimorando e fundamentando o assunto em questão abordado na pesquisa. Para obter os resultados e respostas acerca da problematização apresentada, foi realizada uma pesquisa exploratória se valendo da análise bibliográfica de artigos publicados entre 2000 e 2021 , utilizando as bases de dados de artigos científicos Scielo, Google Acadêmico, ANVISA e CDL, BVS (Biblioteca Virtual em Saúde), com isso foi feito o levantamento de dados contido em cada um dos artigos. Os critérios de inclusão dos estudos foram: artigos em português e inglês, que apresentassem em sua discussão, a utilização da toxina botulínica tipo A para fins terapêuticos. 


\section{Referencial Teórico}

\subsection{Toxina botulínica}

A história da toxina botulínica teve início na década de 90 quando se percebeu a eficácia em pacientes com rugas que foram tratadas contra a ação involuntária dos músculos da pálpebra, tendo como principal mecanismo de ação o bloqueio da acetilcolina na ligação neuromuscular, sendo assim apto a causar a paralisia muscular e assim melhorando os quadros de dores que o paciente se encontra. Atualmente, a toxina botulínica (TBA) é uma das mais importantes substâncias no ramo do rejuvenescimento facial não sendo uma aplicação invasiva, evitando assim meios cirúrgicos. É produzida por uma bactéria grampositiva e anaeróbica, chamada clostridium botulinium (Benecke 2012).

O primeiro registro deste tratamento foi realizado por Alan B. Scott e Edward J. Schantz no início do ano de 1970, quando foi utilizado o soro do tipo A na medicina para corrigir o estrabismo. Foram produzidas e fabricadas outras preparações no Reino Unido, Alemanha e China (Erbguth 2008).

Em relação aos mecanismos de ação da TBA é imprescindível discorrer inicialmente sobre à clássica atuação que inibe a liberação de acetilcolina nos terminas nervosos motores e posteriormente nos demais neurotransmissores, dessa forma, segundo Maria Matilde Sposito (2014) é possível afirmar que os mecanismos de ação do toxina botulínica incluem o relaxamento muscular, a ação antinociceptiva (que inclui o bloqueio da liberação de peptídeos referentes à dor), sistema nervoso autônomo e efeitos diretos e indiretos sobre o sistema nervoso central.

Sabe-se que a substância supracitada possui utilização popularmente conhecida no ramo estético e terapêutico com efeito estético a TBA pode reduzir os danos do envelhecimento com procedimentos não invasivos, todavia, a toxina botulínica tem atuação eficaz em outras áreas e necessidades (Dall'antonia et all, 2013).

Partindo desse pressuposto, este projeto de pesquisa apresentará a exploração da toxina botulínica num quadrante não tão previsível que é no âmbito estético, mas explorará a ministração desse fármaco no tratamento da dor a partir das propriedades analgésicas, pois, conforme dispõe Maria Matilde Sposito (et all, 2014) esta é uma das formas de utilização da referida substância além da tão conhecida função de reversão de linhas de expressão.

O contexto histórico da toxina botulínica tem seu marco inicial em 1817, ano em que houve a primeira publicação descrevendo a toxina como causadora do botulismo, tal reação seria o envenenamento pela Clostridium botulinum, o responsável pela constatação foi o físico alemão Justinius Kerner, segundo ele a presença da substância em salsichas defumadas (botulus salsicha no latim) teria intoxicado diversas pessoas causando a morte delas, segundo o estudioso o ativo agia no Sistema Nervoso Motor e Autônomo, dessa forma propôs uma infinidade de possibilidades controladas da então toxina botulínica na Medicina (Colhado; Boeing, Ortega apud Fujita, 2018).

Já em 1895, houve um novo surto de botulismo na vila belga de Elezelles, para descobrir a causa Emile Van Ermengem estudou os tecidos dos corpos das trinta e quatro pessoas falecidas e encontrou em comum entre eles a presença de uma bactéria presente na crua de porco crua, bactéria esta que foi então associada ao botulismo pelas semelhanças, Van Ermengem para confirmação da suspeita isolou a bactéria e deu o nome de Bacillus botulinus, posteriormente renomeada por Clostridium Botulinum (Quagliato, Barasnevicius, 2014).

Em 1960, o oftalmologista Alan B. Scott buscava uma substância que auxiliasse o tratamento de estrabismo em crianças, assim, descobriu que a toxina botulínica quando inserida nos músculos hiperativos proporcionava a reação que ele procurava (Dressler, 2012).

Todavia, com o passar do tempo a toxina botulínica ganhou espaço em inúmeras áreas com os mais diversos objetivos no âmbito medicinal, em especial na oftalmologia, neurologia e na dermatologia estética, desempenhando, portanto, um papel crucial na história de utilização da referida substância (Silva, 2012). 
A toxina botulínica foi liberada no Brasil em 1997 pelo Ministério da Saúde e Agência Nacional de Vigilância Sanitária, porém se popularizou quando surgiu no mercado o BOTOX®29, como primeira marca do medicamento produzida e comercializada em solo brasileiro. Vale ressaltar que a utilização terapêutica da TBA não pode ser de forma irrestrita, exige-se a realização de ensaios clínicos que tracem diretrizes para seu uso e qualquer afeito adverso deve ser notificado à Gerência de Farmacovigilância desde a sua constatação (Anvisa, 2007).

\subsection{Mecanismos de ação}

A atuação da toxina botulínica pode ser resumida na inibição da liberação exocitótica da acetilcolina em qualquer terminal nervoso o que, por consequência, reduz a contração do músculo. Esse efeito da toxina faz com que ela se torne útil em tanto clínica quanto terapeuticamente (Aoki, 2005).

A partir dessa premissa, a toxina botulínica no tratamento de patologias onde há distúrbios do movimento mostra-se extremamente benéfica inclusive no alívio de dores associadas. Além do mais, como em toda intervenção no organismo, exigiuse a observação de possíveis efeitos colaterais e a relação destes com os pontos onde a toxina botulínica foi injetada, dessa análise adveio a indução ao pensamento de outras possibilidades como a utilização nas glândulas e músculos lisos, assim, foi preciso estender o campo de estudo que envolve os mecanismos de ação relacionados à toxina botulínica (Sposito et all, 2014).

Portanto, é preciso se ater que ao clássico mecanismo de ação acima descrito, seja: a inibição da liberação de acetilcolina nos terminais nervosos motores e outros neurotransmissores, pois, há outros mecanismos que igualmente merecem estudo como o relaxamento muscular abrangendo os músculos estriados e reflexos de estiramento da medula; ação antinociceptiva que impede a liberação de peptídeos ligados à dor; sistema nervoso autônomo que age sobre glândulas salivares, sudoríparas e lacrimais, atividade sobre a bexiga e próstata; efeitos diretos ou não que agem sobre o Sistema Nervoso Central .

\subsection{Duração da ação da Toxina Botulínica tipo A}

Depois de usar a toxina, o efeito é de forma temporária, na maioria dos casos, por cerca de seis meses, após este período, é mais intenso de três a quatro meses, gradativamente após isto o movimento começa a enfraquecer. A toxina começa a funcionar 2 a 5 dias após a aplicação.

Deve-se notar também que o tempo de efeito pode variar conforme o sorotipo e tipo de toxina para o tratamento, geralmente do tipo A, que é a mais utilizada, podendo durar por até seis meses.

Devido à reinervação dos nervos, a função é restabelecida pelas fibras musculares esqueléticas e regeneração proteica do complexo SNARE, levando a acetilcolina a ser liberada e os músculos se contrair novamente (Kanem e Sattlerg, 2016).

\subsection{Utilização da Toxina Botulínica tipo A para fins terapêuticos}

\subsubsection{Enxaqueca}

A enxaqueca é evidente de forma recorrente como um tipo de dor de cabeça intensa, muitas vezes seguida de vômitos e náuseas, devido à intensa dor. A crise acontece normalmente a cada 45 dias e a duração varia entre 24 a 48 horas. Afeta tanto os homens como as mulheres, sendo mais comum nas faixas etárias de 18-29 anos e 40-49 anos, com maior incidência nas mulheres (C Lazzaretti - 2021)

A enxaqueca crônica é caracterizada por ataques de dor de cabeça que ocorrem 15 ou mais vezes no dia por mês. Isto é considerado sério porque é altamente incapacitante, afeta seriamente a qualidade a vida pessoal.

Assim que o diagnóstico de enxaqueca for confirmado, principalmente crônicas, medidas preventivas e tratamentos é fundamental para tentar amenizar ou mesmo prevenir a ocorrência de novas crises. Uma das opções de tratamento aprovadas 
pelo FDA desde 2010 é o uso da TxB tipo A para tratar a enxaqueca crônica. Após a aplicação, TxB tipo A atuará na sensibilização periférica, inibindo a substância P que é composta por nervos sensoriais, glutamato e outros neurotransmissores e neuropeptídios. É responsável por causar excitação das células nervosas e desencadear processos inflamatórios, reduzindo indiretamente a sensibilização central e levando ao alívio da dor. Os músculos utilizados para a aplicação são: prócero, corrugador, frontal, temporal, occipital, trapézio e cervical postero-superior (G de Castro Kraemer - 2021)

Figura 1. Distribuição dos pontos de aplicação da TxB tipo A no tratamento para enxaqueca.
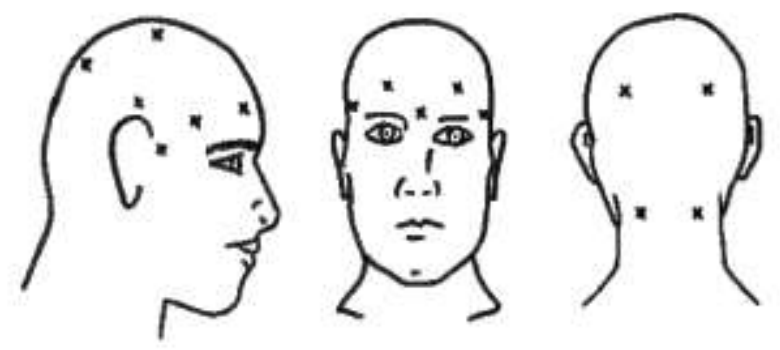

Fonte: Menezes (2007).

\subsubsection{Hiperidrose}

A sudorese é necessária para o equilíbrio da temperatura do corpo, a hiperidrose é definida pelo acumulo da sudorese, que é classificada como hiperidrose primária e secundária, gerada por alterações idiopáticas, se manifestando de forma crônica, propício à hiperatividade do SN autônomo simpática, que leva a hipersecreção das glândulas sudoríparas (TM de Souza- 2019)

O acumulo excessivo da sudorese pode agredir várias regiões, como também pode se manifestar em somente uma, quando ocorre em mais de uma região é chamado hiperidrose associada. As principais regiões de manifestações da hiperidrose são a axila, palmas e plantas dos pés, locais onde são localizadas as glândulas écrinas, a principal glândula sudorípara do corpo. Afeta comumente homens e mulheres, que pode acontecer em todas as faixas etárias, principalmente na adolescência, cerca de 30\% a 50\% dos casos relatam um histórico familiar e o diagnóstico é puramente clínico (CZP Santos - 2018)

O tratamento mais utilizado é o uso de antitranspirantes, mas não é suficiente em determinados casos de hiperidrose, existe o método cirúrgico que realiza a remoção das glândulas sudoríparas de forma definitiva. Um novo método no qual envolve a aplicação de TxB tipo A, está sendo muito manuseada por ser de extrema eficácia, a TxB tipo A age bloqueando provisoriamente a liberação do neurotransmissor acetilcolina, ou evitando que seja liberado na fenda sináptica, e, contudo, a glândula sudorípara passa a não receber estímulo para a secreção. É considerado um método acessível e que pode ser aplicado com anestesia tópica ou local, possui um efeito terapêutico temporário de até 7 meses de duração (RS de Souza -2019)

Para este procedimento é usado uma seringa com agulha fina, onde são aplicadas pequenas doses da toxina próximo a glândula sudorípara, as aplicações são feitas com a distância de 1,5 cm a fim de que todas as glândulas sejam alcançadas. 
Figura 2. Pontos $1 \mathrm{~cm}$ a $2 \mathrm{~cm}$, na região axilar, para injeções intradérmicas de toxina botulínica tipo A



Fonte: Reis (2011).

\subsection{Papel da toxina botulínica no tratamento da dor}

A toxina botulínica é uma substância gênero que se subdivide em sete tipos de toxinas: A, B, C (1 e 2), D, E, F e G, nem todos são comumente agentes causadores do botulismo, essa condição ocorre mais com a reação do organismo ao se deparar com a presença dos tipos A, B, E e F, no tratamento de espasmos, dor e na estética o tipo mais ministrado é o A, visto que ele é o mais potente de todos o que faz com que dozes reduzidas já sejam suficientes para alcançar o efeito desejado (Rowlands et all, 2010).

Segundo estudos sobre o tema, a TBA não apenas relaxa o músculo, mas possui também ação analgésica e antiinflamatória, o que revela, portanto, maior utilidade da toxina em tecidos periféricos (SIM, 2011). Isso se dá porque a toxinas atua nas demais substâncias e neurotransmissores que são ativados quando há uma inflamação como a norepinefrina, substância $\mathrm{P}$, peptídeo relacionado ao gene da calcitonina (PRGC) e o glutamato e na percepção do processo doloroso no organismo (Sposito, 2009).

A TB se mostra uma alternativa segura quanto ao tratamento de síndromes dolorosas, estudos mostram que seu uso vem crescendo na área médica. Várias publicações têm revelado a eficácia e segurança da toxina botulínica no tratamento de algumas síndromes, como Neuralgia do Trigêmeo, Neuralgia Pós-herpética, Síndrome Dolorosa Regional Complexa, Síndrome Dolorosa Miofascial e na Cefaleia (Colhado, 2019).

A neuralgia do trigêmeo (NT) se caracteriza por uma disfunção no nervo de mesmo nome, a qual ocasiona episódios de dores severas e excruciantes. Esta doença é, geralmente, unilateral, sendo que ocorre com maior frequência do lado esquerdo da face. O tratamento convencional da NT baseia-se na utilização de medicamentos anticonvulsivantes, principalmente a base de carbamazepina. Estes possuem como efeitos colaterais sonolência, diplopia e instabilidade, podendo resultar também em comprometimento hepático, sendo necessário acompanhamento periódico através de exames laboratoriais, como hemogramas e provas de função hepática (Frizo, 2004).

Outra condição dolorosa tratável com a toxina botulínica é a neuralgia pós-herpética (NPH), a qual é caracterizada pela dor neuropática persistente distribuída em locais onde houve manifestação prévia de herpes-zoster, sendo que a mesma se manifesta aproximadamente três meses após o desaparecimento das lesões dérmicas do herpes zoster (Spátola, 2010).

O tratamento da NPH é realizado através da administração de fármacos, dentre eles são utilizados os anticonvulsivantes e antidepressivos como tratamento de primeira linha, e os opioides como tratamento de segunda linha, através de bloqueios neurais, estimulação medular ou até mesmo excisão cirúrgica (Portela, 2013). 
Traumatismos, sobrecarga, inflamações e modificações dos tônus musculares podem causar tensões musculares com intensa dor localizada e à distância, essa condição é chamada de síndrome dolorosa miofascial, o uso da TB em pacientes com SDM, tem se mostrado suficiente para uma melhora no quadro de dor crônica apresentada pelos pacientes com essa síndrome. A melhora na qualidade de vida dos pacientes é evidente, quando se compara tratamentos anteriores ao uso da toxina botulínica (Vianna, 2005).

\section{Considerações Finais}

Diante da ampla revisão da literatura realizada neste estudo, pode-se analisar a literatura sobre o uso da toxina botulínica por profissionais especializados, enfatizando a funcionalidade e aplicabilidade do uso da toxina botulínica do tipo A por esses profissionais. Portanto, pode-se concluir que o uso dessa toxina isoladamente ou como procedimento auxiliar tem apresentado avanços consideráveis no contexto de tratamento de mialgias, o que contribui para a melhoria da qualidade de vida de muitas pessoas.

Porém, as especificações e indicações devem ser respeitadas, a posologia aplicada deve ser rigorosamente observada e deve ser realizada por profissionais qualificados. Portanto, não há dúvida de que este trabalho traz informações importantes sobre a utilização da Toxina botulínica e sua aplicabilidade, funcionalidade, com foco no tipo A.

Considerando as questões apresentadas nas publicações e as hipóteses levantadas, faz-se necessário cada vez mais a exploração deste assunto em revisões integrativas, pesquisas bibliográficas e pesquisas de campo para despertar à população científica sobre o efeito benéfico da utilização da toxina botulínica do tipo A para fins terapêuticos no tratamento de mialgias.

\section{Referências}

ANVISA - Agência Nacional de Vigilância Sanitária. Toxinas botulínicas tipo A: ANVISA esclarece questões ligadas à qualidade dos produtos registrados no Brasil, as indicações terapêuticas e a intercambiabilidade entre elas. Brasil, 2007. Acesso em: 19 de abril de 2021.

Aoki, K. R. Review of a proposed mechanism for the antinociceptive action of botulinum toxin type A. Neurotoxicology. 2005.

Carvalho AVC, Gagliani LH. Toxina botulínica tratamento de enxaquecas. Revista UNILUS Ensino e Pesquisa. 2014;11(22):63-76.

Cereser ND, Costa FMR, Rossi Júnior OD, Silva DAR, Sperotto VR. Botulismo de origem alimentar. Ciência Rural. 2008;38(1):280-7

Colhado, O. C. G.; Boeing, M.; Ortega, L. B. Toxina botulínica no tratamento da dor. Brazilian Journal of Anesthesiology, v. 59, n. 3 , p. 366-381, 2019.

Dall'antonia, M.; N., Regina M. de O.; Sanches, M. L.; Guimarães, A. S. Dor miofascial dos músculos da mastigação e toxina botulínica. Revista Dor, v. 14, n. 1, p. 52-57, 2013.

Dressler, D. Clinical applications of botulinum toxin. 2012. Disponível em: Acesso em: 28 de março de 2021.

Gimenez, R. P., Gemperli, R., Salles, A. G., Ferreira, M. C. Análise retrospectiva das alterações das rugas faciais após aplicações seriadas de toxina botulínica tipo A. Revista Brasileira de Cirurgia Plástica. 2010;25(2):297-303

Kreutz, L. M., Heineck, I. O uso da toxina botulínica tipo A na profilaxia da enxaqueca. Trabalho de Conclusão de Curso de Farmácia, Porto Alegre, 2011

Osaki, M. H., Belfort, Júnior, R. Qualidade de vida e custos diretos em pacientes com blefaroespasmo essencial e espasmo hemifacial tratados com toxina botulínicaA. Arquivos Brasileiros de Oftalmologia. 2004;67(1):43-9.

Pepino, L. Aplicação de botox: porque ele virou o desejo das mulheres. Disponível em: Acesso em: 29 de março de 2021.

Portella AVT, Souza LCB, Gomes JMA. Herpes-zóster e neuralgia pós-herpética. Register Dor. 2013

Quagliato, E.; Barasnevicius, M. A. Botulinum toxin? how a poison turned to a fascinating ally against an old adversary. Arquivos de neuro-psiquiatria, v. 72, n. 6, p. 401-402, 2014.

Romero, J. G. de A. José; P., R. B. de N.; Almeida-leite, C. M. Toxina botulínica no tratamento da dor na neuralgia trigeminal: revisão de literatura. BrJP, v. 3, n. 4, p. 366-373, 2020.

Rowlands, R. E. G., Ristori, C. A., Lopes, G. I. S. L., Paula, A. M. R., Sakuma, H., Grigaliunas, R., et al. Botulism in Brazil, 2000-2008: epidemiology, clinical lindinhas and laboratorial diagnosis. Revista do Instituto de Medicina Tropical. 2010. 
Research, Society and Development, v. 10, n. 14, e535101422385, 2021

(CC BY 4.0) | ISSN 2525-3409 | DOI: http://dx.doi.org/10.33448/rsd-v10i14.22385

Silva, J. F. N. da. A aplicação da toxina botulínica e suas complicações. Revisão Bibliográfica. Dissertação (Mestrado em Medicina Legal) - Instituto de Ciências Biomédicas de Abel Salazar - Universidade do Porto. Porto/Portugal, 2012.

Sim, W. S. Application of botulinum toxin in pain management. The Korean Journal of Pain. 2011.

Spátola, A. Neuralgia pós-herpética-tratamento da dor neuropática com uso da toxina botulínica tipo A-apresentação de um caso. Revista Medicina de Reabilitação. 2010

Sposito, M. M. de M. Toxina Botulínica do Tipo A: mecanismo de ação. Acta de fisiátrica. 2009.

Sposito, M. M. de M.; T., Stephanie, A. F. Toxina Botulínica Tipo A no tratamento da dor miofascial relacionada aos músculos da mastigação. Acta fisiátrica, 2014.

Unno, E. K., Sakata, R. K., Hissy, A. M. Estudo comparativo entre toxina botulínica e bupivacaína para infiltração de pontos-gatilho em síndrome miofascial crônica. Revista Brasileira de Anestesiologia. 2005.

Vianna, V. R. Tratamento da dor Miofascial com o emprego de toxina botulínica. Dissertação (Especialização em Disfunção Temporomandibular e Dor Orofacial), Universidade Federal de Santa Catarina, Florianópolis, 2005. 